\title{
Modeling and Analysis of a Visual Feedback System to Support Efficient Object Grasping of an EMG-Controlled Prosthetic Hand
}

\begin{abstract}
Millions of people around the world have lost their upper limbs mainly due to accidents and wars. Recently in the Middle East, the demand for prosthetic limbs has increased dramatically due to ongoing wars in the region. Commercially available prosthetic limbs are expensive while the most economical method available for controlling prosthetic limbs is the Electromyography (EMG). Researchers on EMG-controlled prosthetic limbs are facing several challenges, which include efficiency problems in terms of functionality especially in prosthetic hands. A major issue that needs to be solved is the fact that currently available low-cost EMG-controlled prosthetic hands cannot enable the user to grasp various types of objects in various shapes, and cannot provide the efficient use of the object by deciding the necessary hand gesture. In this paper, a computer vision-based mechanism is proposed with the purpose of detecting and recognizing objects and applying optimal hand gesture through visual feedback. The objects are classified into groups and the optimal hand gesture to grasp and use the targeted object that is most efficient for the user is implemented. A simulation model of the human hand kinematics is developed for simulation tests to reveal the efficacy of the proposed method. 80 different types of objects are detected, recognized, and classified for simulation tests, which can be realized by using two electrodes supplying the input to perform the action. Simulation results reveal the performance of proposed EMG-controlled prosthetic hand in maintaining optimal hand gestures in computer environment. Results are promising to help disabled people handle and use objects more efficiently without higher costs.
\end{abstract}

Keywords: Prosthetic Hand, Electromyography, Object Detection, Objects Grasping.

https://doi.org/10.1515/cdbme-2019-0053

\footnotetext{
*Corresponding author: Tolgay Kara: Gaziantep University, Dept. Electrical and Electronics Eng. Gaziantep TURKEY, kara@gantep.edu.tr

Ahmad Soliman Masri: Gaziantep University, Dept. Electrical and Electronics Eng. Gaziantep TURKEY
}

\section{Introduction}

Commercially available prosthetic limbs are expensive because of costly technologies and materials in addition to expertise needed for their manufacturing. Basically the prosthetic limbs can be classified depending on installation mechanism into three main classes: Prosthetic limbs that are surgically installed are directly connected to the nerve endings. The sensors can read more signals therefore provide more functionality. They are more expensive due the costly technologies. Another class is installed without surgery using Electroencephalography (EEG). They make use of sensors that can read and record the electrical activity of the brain to be translated at the end to an action, which can be performed by the prosthetic limbs. They are less expensive than surgical limbs. Third class of prosthetic limbs is installed without surgery using EMG. They use sensors that can evaluate and record the electrical activity produced by skeletal muscles. They are cheaper but with less functionality. Moreover, these limbs are limited in terms of customization options of shape, size and functionality which can cause mental confusion. Because of these limitations, only a small fraction of people is able to use prosthetic limbs. This paper is a part of a multistage research that aims to enable much wider access to prosthetic limbs and produce low cost and highly customizable prosthetic limbs that can be used more efficiently. Design and simulation of the proposed visual feedback system to improve efficiency in object grasping constitutes the main goal of this paper. Implementation of proposed method on EMG based prosthetic hands is a future goal of the study.

\subsection{Problem definition}

Due to the number of input signals that can be obtained from the muscles using the EMG without causing a mental confusion, it is possible to perform only two actions with the manipulated limb, which are either opening the hand or closing it using all five fingers. As a result, the user can only grasp limited types of objects and cannot use them properly. 
Therefore, it is important to use a mechanism other than EMG to detect and recognize the target object and provide the necessary feedback for determining the optimal action to grasp the object in the most efficient way.

\section{Proposed solution}

Based on the limited commands and control signals that can be driven and distinguished from the muscles using EMG without causing mental confusion, the prosthetic hand must become more intelligent and able to understand the surrounding environment so that it can perform more tasks. Our idea is to improve the two EMG based hand and extend its functionality by providing a feedback to the control loop so that it can understand the environment around it and perform its actions according to the targeted object. The proposed solution assumes that the user has no vision impairment and is able to operate the EMG based prosthetic hand without any additional assistance, which means the user can orient the arm properly in approaching the object to be grasped.

In order to find a solution and an appropriate method, we started our research with the analysis of hand movement and human behaviour in dealing with objects. The results of our research with objects and their use depend primarily on the knowledge of the characteristics such as shape and dimensions, and extend to the optimal way to grasp and use it acquired by the accumulated human experience. We also found that human decision in grasping depends mainly on the sense of vision and touch in order to recognize the properties of objects. When a similar object has been dealt with previously, information and the required experience can be considered to be available. As soon as the object is seen, the nature of the object and its dimensions are determined and the decision is taken so that the hand fingers are pre-shaped in order to grasp it. The properties and the success of the grasping process are confirmed by the tactile and visual senses. In our case (EMG based prosthetic hand) the user can see the object but cannot control the fingers in the prosthetic hand for pre-shaping and complete the task due the limited number of input signals acquired from the muscles without causing a mental confusion. The idea is incorporating a vision system that can recognize the object and give orders to the fingers for self-pre-shaping to grasp it properly. This has lead us to the proposed solution in this paper, which is using the computer vision that feeds the system with the necessary information, which helps it make the right decision in order to let the prosthetic hand take the appropriate position to complete the required task.

\section{Methods and implementation}

The proposed solution has four stages. The first stage is using image processing algorithms for object recognition and detecting physical properties of the object. The second stage the grasp analysis for determining how many fingers are needed to grasp the detected object based on its properties. The third stage is using the mathematical modelling and computer simulation techniques for testing the proposed approach on a simulation model of the prosthetic hand. The last stage is the discussion of the simulation tests and improvements.

\subsection{Object recognition}

In our prototype, we use a webcam to feed the prosthetic hand system with the live stream of the surrounding environment. We have used OpenCv with Python to implement the object recognition stage for our proposed solution on top of TensorFlow Object Detection Application Programming Interface (API). We depend on the first version of mobilenet [1] as an efficient convolutional neural network for mobile vision applications with its pre-trained model on coco dataset, which is able to detect and recognize 80 deferent types of objects. We have selected the nearest object to the centre in the scenes that contain multiple objects.

\subsection{Grasp planning}

Our purpose in this stage is to select grasp type which fits best the recognized object. We have started by studying the grasp types and we found that a lot of researchers such as [2, 3] have studied the grasp operation for recognizing the possible grasp types and categorize them based on functionality and use. We have selected nine grasping types which have a high frequency of daily usage based on [4] and able to cover different functionality, object shape, and finger positions. In this paper we select the appropriate grasp type out of the nine grasp types which fits the recognized object based on the object attributes that we extract on the previous stage. We depend in this approach on a work carried out by Cai et al. in 2016 [5], in which the authors have categorized the objects based on four attributes according to shape (Prismatic, Round, Flat) and rigidity (deformable or not). It is presented in Figure 1 an example of objects according to their attributes.

The way that three different shape classes are recognized based on the criteria is presented in Table 1 while the fourth attribute of deformability can be determined in terms of 

Hand -209

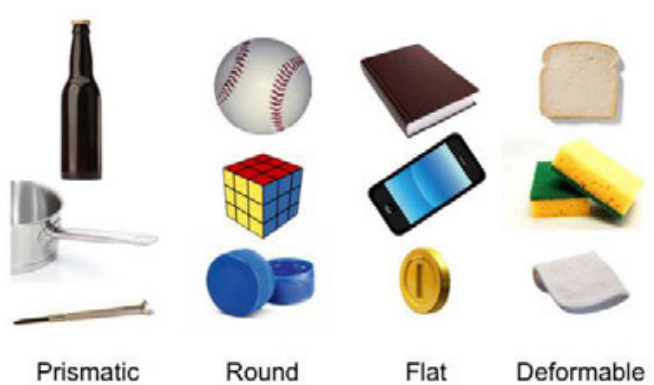

Figure 1: Object examples with four different attributes: Prismatic, Round, Flat, and Deformable [5].

whether the object deforms under normal grasping forces. So, based on these object shape classifications and required functionality (Power and Precision) of the recognized object we can select one of the nine grasp types presented in Figure 2. Finally, we have assigned the 80 objects to the corresponding grasp types based on the mentioned method.

Table 1: Classification criterion of three shape classes. Length of object along three object dimensions (major axes of the object) are denoted as $A, B$, and $C$, where $A \geq B \geq C$.

\begin{tabular}{cc}
\hline Shape classes & Object dimensions \\
\hline Prismatic & $A>2 B$ \\
Round & $B \leq A<2 B, C \leq A<2$ C \\
Flat & $B>2 C$ \\
\hline
\end{tabular}

\subsection{Hand modelling}

The modelling part is based on SynGrasp, which is a toolbox developed for the analysis of grasping, suitable both for robotic and human hands [6]. The hand structure is defined in terms of fingers, links and joints. A cell named base, containing as many elements as the number of fingers, collects in each cell element a $4 \times 4$ matrix representing the homogeneous transformation matrix between the wrist reference frame and a reference frame defined at the beginning of each finger kinematic chain. Denavitt-

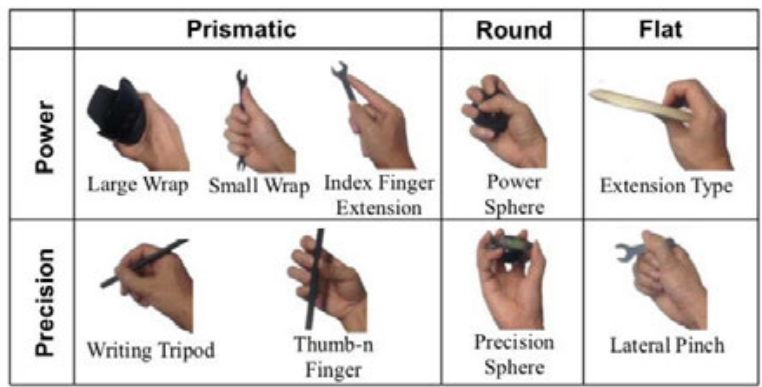

Figure 2: Nine grasp types selected from [3], grouped by functionality (Power and Precision) and object shape (Prismatic. Round and Flat).
Hartenberg (DH) parameters have been chosen as default notation. A table containing the $\mathrm{DH}$ parameters of each finger has to be provided to describe a hand. A cell named DHpars, which has as many elements as the number of fingers, which is five in our case, collects in each element a matrix with four columns and as many rows as the number of joints of each finger four joints in our case. Each row represents the $\mathrm{DH}$ parameters that allow defining the joint with respect to the preceding one or with respect to the base reference frame. For each hand finger, a structure is created with the function SGmakefinger, taking as input the $4 \times 4$ homogeneous matrix base, the dhpar matrix containing the $\mathrm{DH}$ parameter table, and the vector $\mathrm{q}$ of joint variables, where dhpar and $q$ have as many rows as the number of joints of the finger. After defining the hand structure, we have provided the joint variables for the nine types of grasping. Results are presented in Figure 3.

\section{Simulation results}

Our project performance is primarily dependent upon the object recognition algorithm performance. In this paper we have used the latest ('2017_11_17') pre-trained model on coco dataset. It is based on the first version of mobilenet that is able to detect and recognized an object from coco dataset on $30 \mathrm{~ms}$ as an average based on MSCOCO evaluation protocol and with $21 \mathrm{mAP}$ which is computing the interpolated average precision (AP) for each class and averages it among all classes (mAP). Figure 4 provides snapshots of captured images by webcam, and proposed system successfully identifying the correct grasp type for 8 objects which we perform the test on. In each case, an object has been placed in front of the webcam that captured an image to classify the correct grasp, which is simulated in the right hand side of the snapshots. All eight grasps are represented, one per row. The videos along with additional real-time experiments, can be found at [7].

\section{Conclusion}

In this paper, we propose a system for efficient object grasping of an EMG-controlled prosthetic hand by visual feedback, which is an optimal solution for overcoming the limitations of the EMG based hand. Proposed system can especially be useful in grasping 80 objects from coco dataset. We have used TensorFlow Object Detection API to detect and recognize multiple objects, then we used Python to 
T. Kara et al., Modeling and Analysis of a Visual Feedback System to Support Efficient Object Grasping of an EMG-Controlled Prosthetic Hand -210
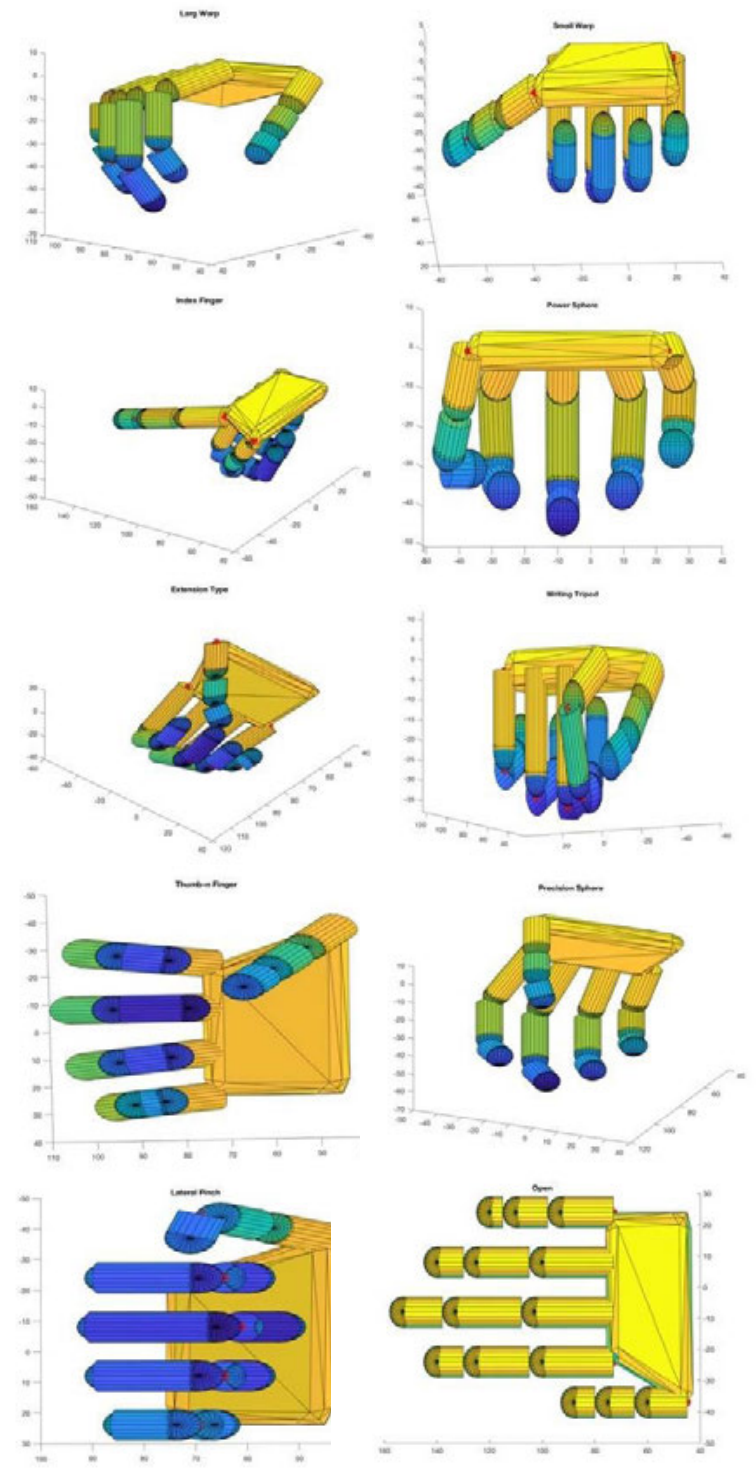

Figure 3: Modeling results for the nine grasping types and open hand.

classify them in groups and propose the optimal posture to grasp/use the targeted object in a way that can be most useful to the user. Finally, we have simulated the optimal posture for the hand in computer environment and revealed the performance of proposed method. Results are promising for implementation of proposed method in real EMG-controlled prosthetic hand applications. Future goal of the study is constructing a prototype of proposed prosthetic hand design and applying learning algorithms to improve its performance.

\section{Author Statement}

Research funding: The author state no funding involved. Conflict of interest: Authors state no conflict of interest. Informed consent: Informed consent has been obtained from all individuals included in this study. Ethical approval: The research related to human use complies with all the relevant national regulations, institutional policies and was performed

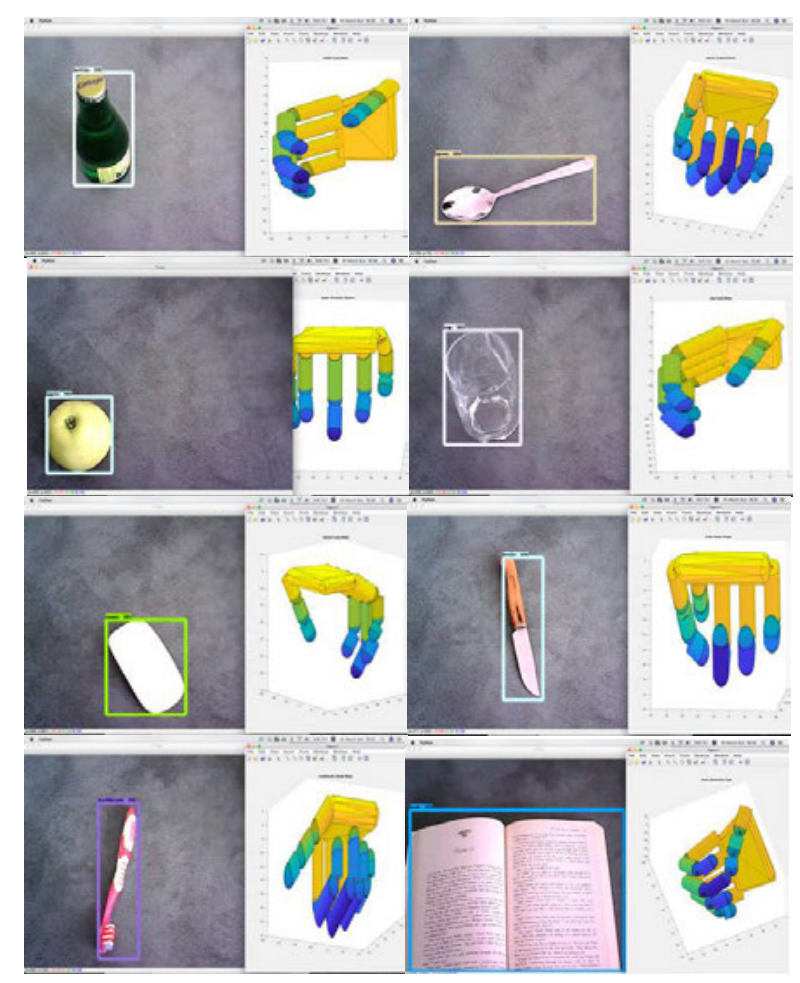

Figure 4: Snapshots of captured images, proposed system recognizes the objects and select the correct grasp types successfully.

in accordance with the tenets of the Helsinki Declaration, and has been approved by the authors' institutional review board or equivalent committee.

\section{References}

[1] Howard AG, Zhu M, Chen B, Kalenichenko D, Wang W, Weyand T, Andreetto M, Adam H. Mobilenets: Efficient convolutional neural networks for mobile vision applications. arXiv preprint arXiv:1704.04861. 2017 Apr 17.arXiv: 1704.04861

[2] Cutkosky MR. On grasp choice, grasp models, and the design of hands for manufacturing tasks. IEEE Transactions on robotics and automation. 1989 Jun 1;5(3):269-79.

[3] Feix T, Pawlik R, Schmiedmayer HB, Romero J, Kragic D. A comprehensive grasp taxonomy. InRobotics, science and systems: workshop on understanding the human hand for advancing robotic manipulation 2009 Jun 28 (Vol. 2, No. 2.3, pp. 2-3).

[4] Bullock IM, Zheng JZ, De La Rosa S, Guertler C, Dollar AM. Grasp frequency and usage in daily household and machine shop tasks. IEEE transactions on haptics. 2013 Jul;6(3):296308.

[5] Cai M, Kitani KM, Sato Y. Understanding Hand-Object Manipulation with Grasp Types and Object Attributes. InRobotics: Science and Systems 2016 Jun 18 (Vol. 3).

[6] Malvezzi M, Gioioso G, Salvietti G, Prattichizzo D. Syngrasp: A matlab toolbox for underactuated and compliant hands. IEEE Robotics \& Automation Magazine. 2015 Dec;22(4):5268.

[7] "Test.mov." Google Drive, https://goo.gl/51mWRH. 